\title{
The Supernovae Analysis Application (SNAP)
}

\author{
Amanda J. Bayless ${ }^{1}(\mathbb{1})$, Chris L. Fryer ${ }^{2,3,4}$ (1) , Ryan Wollaeger $^{2}$, Brandon Wiggins ${ }^{2,5}$ (1), Wesley Even ${ }^{2}$ (1), Janie de la Rosa ${ }^{6}$, \\ Peter W. A. Roming ${ }^{1,6}\left(\mathbb{0}\right.$, Lucy Frey ${ }^{2}$, Patrick A. Young ${ }^{7}(1)$, Rob Thorpe ${ }^{1}$, Luke Powell ${ }^{1}$, Rachel Landers ${ }^{1}$, \\ Heather D. Persson ${ }^{1}$, and Rebecca Hay ${ }^{1}$ \\ ${ }^{1}$ Southwest Research Institute, Department of Space Science, 6220 Culebra Road, San Antonio, TX 78238, USA; abayless@ @wri.edu \\ ${ }^{2}$ Los Alamos National Laboratory, Los Alamos, NM 87545, USA \\ ${ }^{3}$ Physics Department, University of Arizona, Tucson, AZ 85721, USA \\ ${ }^{4}$ Physics and Astronomy Department, University of New Mexico, Albuquerque, NM 87131, USA \\ ${ }^{5}$ Southern Utah University, $351 \mathrm{~W}$ University Boulevard, Cedar City, UT 84720, USA \\ ${ }^{6}$ University of Texas at San Antonio, San Antonio, TX 78249, USA \\ ${ }^{7}$ School of Earth and Space Exploration, Arizona State University, 411 North Central Avenue, Phoenix, AZ 85004, USA \\ Received 2016 July 1; revised 2017 July 27; accepted 2017 July 29; published 2017 September 6
}

\begin{abstract}
The SuperNovae Analysis aPplication (SNAP) is a new tool for the analysis of SN observations and validation of SN models. SNAP consists of a publicly available relational database with observational light curve, theoretical light curve, and correlation table sets with statistical comparison software, and a web interface available to the community. The theoretical models are intended to span a gridded range of parameter space. The goal is to have users upload new SN models or new SN observations and run the comparison software to determine correlations via the website. There are problems looming on the horizon that SNAP is beginning to solve. For example, large surveys will discover thousands of SNe annually. Frequently, the parameter space of a new SN event is unbounded. SNAP will be a resource to constrain parameters and determine if an event needs follow-up without spending resources to create new light curve models from scratch. Second, there is no rapidly available, systematic way to determine degeneracies between parameters, or even what physics is needed to model a realistic SN. The correlations made within the SNAP system are beginning to solve these problems.
\end{abstract}

Key words: catalogs - radiation mechanisms: general - supernovae: general

\section{Introduction}

One of the most crucial processes for shaping the composition of the universe is the death of massive stars manifest as supernovae $(\mathrm{SNe})$. Understanding this process is important as it has significant impacts on our understanding of cosmology, chemical enrichment, galaxy evolution star formation rate, stellar evolution, compact object remnants, the circumstellar medium, and dust formation. SNe also serve as laboratories of high-energy, high-density regimes, which cannot be probed effectively by experiments on Earth.

$\mathrm{SNe}$ are classified by the evolution of their emission and spectral features (for reviews, see Filippenko 1997, 2005) with Types Ib, Ic, and II being believed to arise from the collapse of the core of a massive star and subsequent stellar explosion. Observational differences between core-collapse events likely arise from either variations in the progenitor star or the circumstellar environment, or possibly characteristics of the explosion mechanism itself (e.g., Arnett 1996; Heger et al. 2003). Multiple code bases (e.g., Blinnikov \& Popov 1993; Dessart \& Hillier 2005; Kasen et al. 2006; Frey et al. 2013a; Wollaeger et al. 2013) are solely dedicated to modeling the evolution of SN luminosity or SN light curves, and recently efforts have begun to make detailed numerical comparisons of these codes (Kozyreva et al. 2017). Previously, interesting SNe have been modeled individually (e.g., Dessart et al. 2015; Chatzopoulos et al. 2016) and such studies have provided constraints on, e.g., mass loss and stellar evolution (Frey et al. 2013a; Bayless et al. 2015) or insights into the explosion mechanism itself (Grefenstette et al. 2014). Calculating emission can be an involved process, in some cases requiring $\sim 10,000$ CPU hours per light curve, with large suites of simulations dedicated to studying a single explosion (Frey et al. 2013a).

Upcoming instruments, including the LSST, will open the era of large SN surveys in which $>100,000 \mathrm{SNe}$ of all types, core-collapse and Type Ia, will be discovered annually (Valenti et al. 2012). To process this flood of SN data, it becomes imperative to have a means of quickly and robustly characterizing events. This wealth of data will include previously rare or exotic explosions, and to capitalize on these events it will be essential to robustly identify them for extended follow-up. On-the-fly, detailed numeric modeling for every event is impractical, yet very high-fidelity models which include shock radiation hydrodynamics or detailed non-LTE radiative transfer may still be needed to identify SNe exhibiting a particular process of interest. These $\mathrm{SN}$ data will also provide a possible means of validating SN light curve codes over very large parameter spaces, provided each observed SN can be studied inexpensively. Packages for fitting and characterizing $\mathrm{SNe}$ exist in the literature (e.g., Burns et al. 2011), but such applications make a priori assumptions about light curve shape or the nature of the power source. There is also an existing archive for SN observations (Guillochon et al. 2017), but it does not contain models or any method of correlation. In this paper, we present the development of the SuperNovae Analysis aPplication (SNAP), an on-line resource including a database of high-fidelity SN light curve models for characterizing and studying observed SNe.

Swift provides UV light curves of $\mathrm{SNe}$, which are a critical proving ground for SN light curve models (Pritchard et al. 2014). Lack of UV early-time observations causes an underestimation in the bolometric light curve by $\sim 20 \%$ and up to $50 \%$. Fortunately, the Swift UV/Optical Telescope (UVOT; 
Roming et al. 2000, 2004; Roming et al. 2005) has over 50 core-collapse SNe (CCSNe) within its archive (Pritchard et al. 2014) and forms the backbone of the current observational database. At the time of this publication, we are in the process of adding additional Swift $\mathrm{SNe}$ and parsing through groundbased observations of the Swift observed SNe located in the Guillochon et al. (2017) archive. The model portion of the database is currently populated with models from the Los Alamos National Laboratory (LANL, Frey et al. 2013a) and TYCHO (Young \& Arnett 2005).

Ultimately, we would like a unified understanding of how CCSN sub-types are related. This work represents a substantial contribution to this end. In Bayless et al. (2015), we show that the transition between the classical hydrogen-dominated $\mathrm{SNe}$ and hydrogen-poor SNe can be modeled by altering the mass of hydrogen in the outer shell of the star prior to explosion. That study, however, did not fully explore the rich variety of CCSN events. This study brings together for the first time a large array of high-fidelity SN models and a robust means of correlating SN observables with computer simulations. This allows for exploration of a wider range of CCSN events on an unprecedented scale. In this paper, we focus on CCSNe, but we also acknowledge the need for correlating observables for SNe Ia. We are beginning to add Type Ia observations to the database and will have LANL Ia models available in an updated version of SNAP. We hope SNAP will be a community tool, and invite others to add models (CCSNe and Ia).

This paper is structured as follows. In Section 2, we describe the SN database, including the archived observations and numerical light curve models. In Section 3, we detail our method of determining matches and correlations between models and observations. The web tool and interface are described in Section 4 and we conclude in Section 5.

\section{The Supernovae Databases}

The SNAP software consists of four main components: (a) a relational database of models, observations, and correlations, (b) correlation software to compare models and observations, (c) a web application, and (d) software functions to export observations and models and import correlations between observations and models. The database is the most important component of the application, providing a fully relational, constraint-verified database structure for observations, models, and correlations. Figure 1 provides a high-level outline of the database structure. The three main tables (Observations, Models, and Correlations) listed support SNAP science functions, but do not include system-level tables for managing users, recording audit records, etc. The Observations table contains the observational data from the archives as light curve data sets. The Models table contains the synthetic time series spectra and light curves from the LANL modeling code. The Correlations table is where the comparisons between the observations and models are stored. The analysis will be in determining which data-model associations are scientifically interesting.

The SNAP database is implemented in a MySQL relational database that contains 20 custom-designed tables that easily scale and support hundreds of thousands of observations, models, and correlations. The web application functions come from the SwRI template web application, which provides prebuilt web application capabilities such as user management, login authentication, database access, and many other functions. This template web application has been used for dozens of missions including the Juno Science Operations Center, Cassini Ground Data Systems, and New Horizons-Ralph. The web application uses Java as the source software language, and runs on the publicly available Apache web server and Tomcat servlet container. The I/O functions for observations, models and correlations are also being developed in Java; they currently run as stand-alone programs in the first version of SNAP, and will be fully integrated into SNAP in future versions.

\subsection{The Archived Light Curve Observations}

SNAP version 1.0 contains a subset of observations from the Swift Observatory (Gehrels et al. 2004). We are updating SNAP as observations become available and processed into the SNAP formatting. These include more Swift observations and light curves from other telescopes, both space and ground based, many of which are archived in Guillochon et al. (2017). We note that the difference between SNAP and The Open Supernovae Catalog is that SNAP also includes models and a way to relate models and observations, and is not a repository for observations only.

The Swift Observatory includes the UVOT, which contains six filters ranging from the UV to the optical (Poole et al. 2008; Breeveld et al. 2011). The Swift archive is public, but recently the well observed UVOT SN light curves were published in a compilation paper (Pritchard et al. 2014). This work demonstrated that UV emission dominates the bolometric light curve up to 50 days post-explosion. Therefore, including the UV data will be imperative to creating accurate models. There are generally multiple observations of the same SN event with which to correlate models to data and all of the available observations for an event are being compiled within SNAP. Future SN light curves can immediately be input into SNAP and run with the fitting program against available light curve models to produce an instant estimation regarding the nature of the progenitor star and the explosion itself, and a realistic assessment of the error in that estimation. As more SN models are added to the SNAP database the estimation in parameter space will improve. The benefit is that new SNe can be immediately compared to an existing model set, giving an estimate of parameter values, and allowing for a determination of which new SNe should have follow-up observations.

\subsection{The Light Curve Models}

SNAP 1.0 has been optimized for LANL SN models, described below. However, the models are defined by several key parameters (e.g., progenitor mass, metallicity, explosion energy, etc.) that are fairly universal to all models. Thus, SNAP has the ability to host and track most model systems. In the current version of SNAP, a light curve can be uploaded in the form of time series spectra. Code is available to convert the spectra into Swift band light curves for correlations with observations. Future versions of SNAP will include other standard band passes. We note that the database stores only the resulting light curves/spectra and star/explosion parameters. We do not store codes, only the modeling outputs. We also recognize that even with the same parameters, different models can produce different results. This feature of SNAP allows theoretical groups to compare light curves from the same sets 


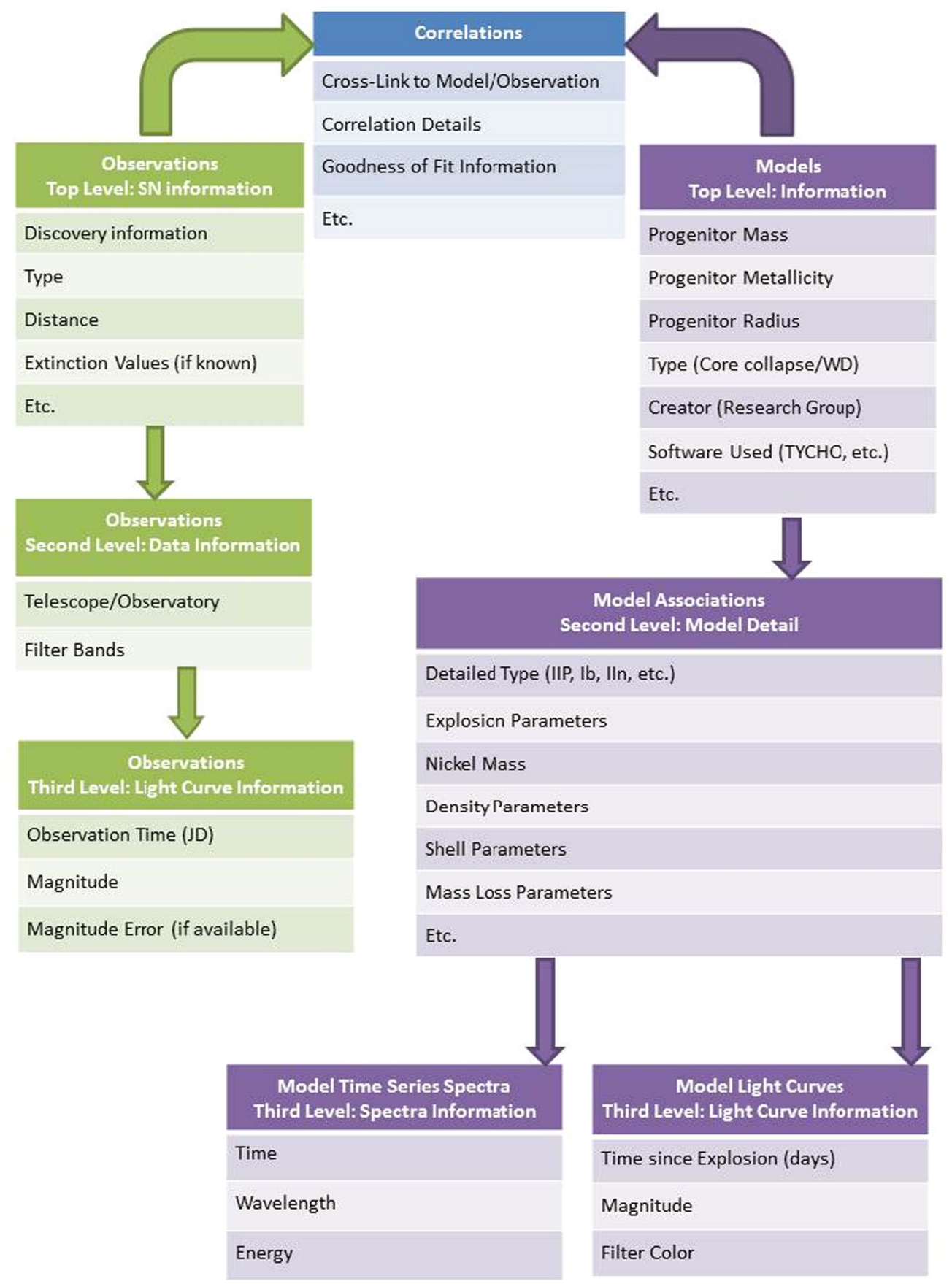

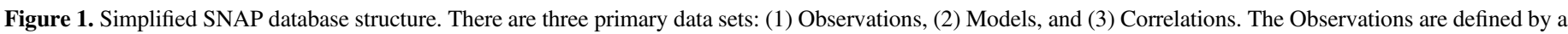

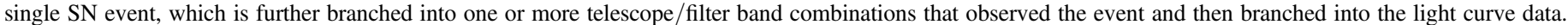

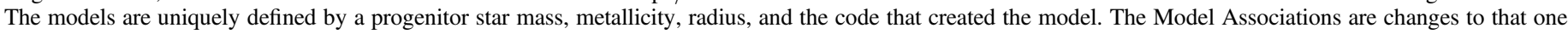

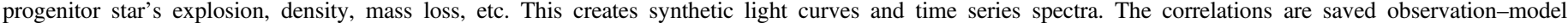
comparisons that were deemed interesting.

of parameters. The database has parameters giving the names of various codes used to make the model and include "Stellar Evolution Software," "Explosion Software," "Explosion Evolution Software," and "Synthetic Spectra Software." Each entry in the database also has an open notes field to include any other information. More information regarding the LANL numerical model can be found in Frey et al. (2013a) and at https:// ccsweb.lanl.gov/astro/transients/data/.

We currently have two modeling methods used for SNAP, both from LANL. The first set of codes are described in detail in Frey et al. (2013a) and summarized here. First, the initial parameters are chosen and a progenitor star is generated using a stellar evolution code (TYCHO; Young \& Arnett 2005). The TYCHO progenitor stars are derived with a new mixing algorithm (very different from the standard mixing length theory) based on solutions of the hydrodymanic equations of convection (Meakin \& Arnett 2007; Arnett et al. 2009; Arnett $\&$ Meakin 2011). TYCHO also includes the options of large mass loss, e.g., a Wolf-Rayet star (Lamers \& Nugis 2002) or binary interaction, which will be needed for the SN Type Ib/c 
analysis. This new algorithm can produce different cores that contain very little helium (Frey et al. 2013b). These cores can drastically change the light curve predictions, particularly in the stripped-core SNe. The explosion and nuclear burning is modeled in a 1D Lagrangian code developed by Herant et al. (1994). This code includes three-flavor neutrino transport using a flux-limited diffusion calculation and a coupled set of equations of state to model the wide range of densities in the collapse phase (see Herant et al. 1994; Fryer et al. 1999 for details).

Evolution of SNe is done with the code Radiation Adaptive Grid Eulerian (RAGE; Gittings et al. 2008). The model from the 1D Lagrangian is mapped into RAGE where the material and radiation are simulated as they push out through the outer layers of the star and into the interstellar medium. RAGE is a multi-dimensional Eulerian radiation-hydrodynamics code with adaptive mesh refinement that can be used to model the physics in a wide range of $\mathrm{SN}$ sub-types. One of the issues in modeling SNe lies in understanding the role of mass loss on the SN light curve, especially if the mass loss is episodic. Interaction of the SN shock with this mass loss will play an important role in modeling SN light curves. RAGE is ideally suited for such situations. It is also a two-temperature model with separate temperatures for radiation and matter. As the simulation evolves, the grid mesh is increased to accommodate the ejecta expansion.

The hydrodynamic models are then post-processed with a separate code, SPECTRUM, to produce synthetic spectra. SPECTRUM calculates luminosity using opacity data derived from the LANL OPLIB database (Magee et al. 1995), which contains data for a grid of 14,900 frequencies. The 1D RAGE output is mapped onto a 2D grid of radial and angular bins. The luminosity of a grid element for a line of sight is determined by the blackbody temperature and the absorption, wavelengthdependent opacity, which is then attenuated by the optical depth. The SPECTRUM code also accounts for the Doppler shifting of photons as they travel outward. Thus, the absorption opacity is determined from Doppler-shifted wavelengths. Due to the lack of readily available models, many observers derive their first estimates of SN progenitor properties using a simple blackbody fit to the observed spectra (see Roming et al. 2012), assuming a single temperature and radius for the emitting surface. Frey et al. (2013a) clearly illustrate the inaccuracy of this assumption. Variations in temperature, density, and composition with radius result in much more complex spectra and light curves than can be calculated using blackbody approximations. Once the spectrum is computed for several given simulation times, a synthetic light curve is computed for a given wavelength bin by applying a filter transmission curve to the spectrum.

The second set of codes use more simplified physics assuming a homologous outflow that models the transport in the gray diffusion approximation (de la Rosa et al. 2016). While this model is more simplistic, it is used because of its computational speed, and thus has the ability to rapidly create light curves for many models. This also is useful to set parameter limitations before running the more elaborate code above. To calculate bands under this approximation, we assume the emission of each zone is a blackbody and calculate the escape fraction of each zone near the photosphere to calculate a broad spectrum. Because we do not include line features (more important at short wavelengths), this method

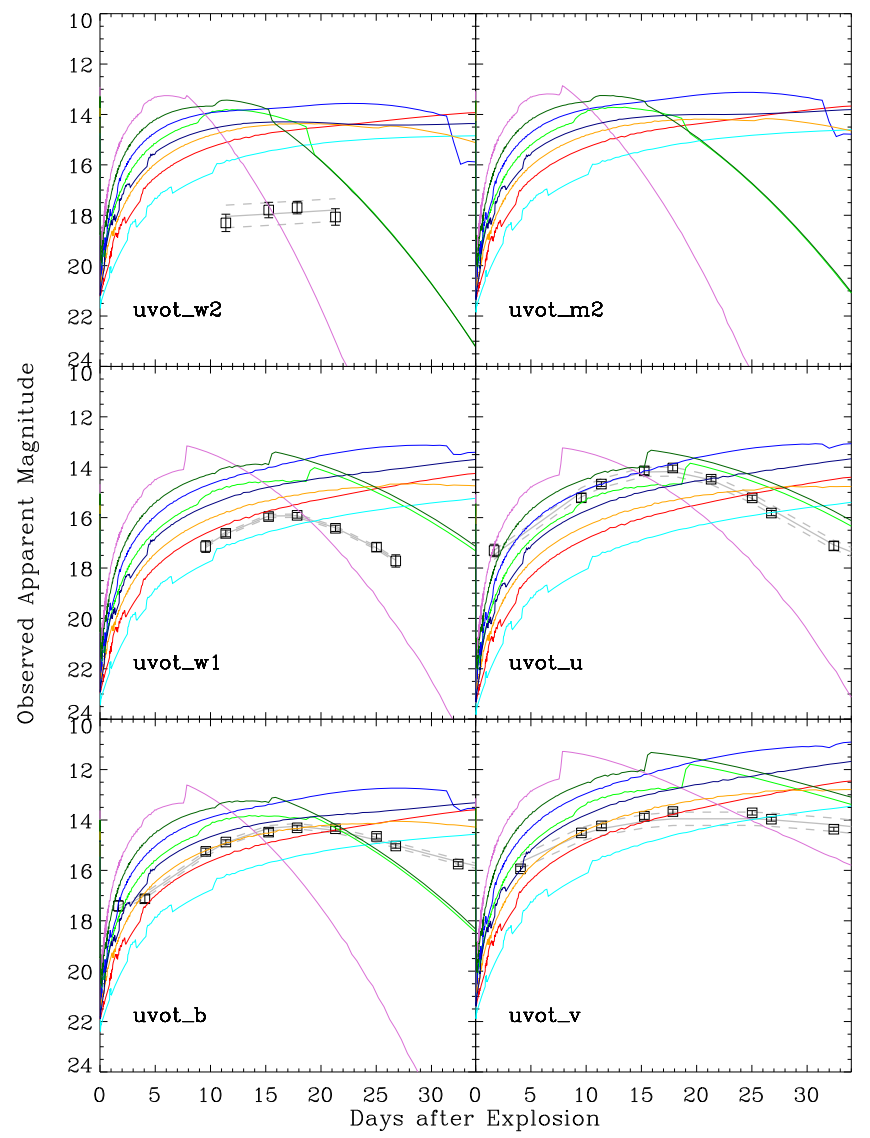

Figure 2. Example of the Gaussian process correlation using the light curve models from de la Rosa et al. (2016). The open squares are SN 2008ax, a type $\mathrm{IIb}$, from the optical Swift-UVOT band passes. The gray solid line is the Gaussian process fit to the observations and the gray dashed lines are the error in the Gaussian fit. The colored lines are a set of models described in the text. This model uses a blackbody spectrum only and thus overestimates the UV as there is no line-blanketing. However, these models are useful as a first approximation of parameter space and their rapid computational time.

will overestimate the emission in the UV and soft X-ray bands of Swift (see Figure 2). Despite its weaknesses, this method is quick enough to calculate large grids of stars, varying the explosion energy, nickel yield, stellar mass, and radius. For this study, we varied the energy ( 1 and 6 foe), explosion mass loss $\left(2,4,6\right.$, and $\left.8 M_{\odot}\right)$, stellar radius (1e12 and $\left.1 \mathrm{e} 13 \mathrm{~cm}\right)$, density profile $\left(r^{0}\right.$ and $\left.r^{-2.5}\right)$, and temperature (ranging from 10 million to 1 billion $\mathrm{K})$. The nickel yield was fixed at $0.1 M_{\odot}$ and the progenitor star in all cases had a zero-age main sequence mass of $25 M_{\odot}$ (prior to mass loss).

At the time of this publication, we are also working on alternative models using either the smooth particle hydrodynamics code SNSPH (Fryer et al. 2006) and the Monte Carlo semi-relativistic thermal radiative transfer code SuperNu (Wollaeger et al. 2013; Wollaeger \& Van Rossum 2014). SuperNu employs a hybrid of Implicit Monte Carlo (IMC, Fleck \& Cummings 1971; Wollaber 2015) for transport and Discrete Diffusion Monte Carlo (DDMC, Densmore et al. 2007, 2012; Abdikamalov et al. 2012) to accelerate transport in optically thick regions of space and photon wavelength. For SuperNu, the expansion is assumed to be homologous (see, for instance, Kasen et al. 2006). The mapping is performed when the ejecta is homologous (when the particles are in a state of ballistic expansion). In SNAP, these multi-dimensional light 
curves could allow observers and theorists to examine which viewing angles of a model best match an observation.

\section{Determining Best Correlations}

The correlations are done by either comparing one model to the observations in the database or one observation (archived or new) to the suite of models in the database. We have several options for correlating models and observations. One option uses the IDL GAUSSFIT library (see the Appendix) to do either a global goodness of fit or to focus on only certain regions of the light curve by specifying a region of time to study. If there are four or more data points in the light curve, the IDL GAUSSFIT works well as it requires a minimum of three degrees of freedom. However, if there are fewer than four data points in the selected region of the light curve, we fit a simple polynomial. We then account for distance and extinction and convert the model flux into the apparent magnitudes. We use published values of the distance to the $\mathrm{SNe}$ and values for the $E(B-V)$ line-of-sight of the host galaxy (if available) from the literature on the particular SN event. The line-of-sight Milky Way extinction comes from Sloan Digital Sky Survey spectra of stars (Schlafly \& Finkbeiner 2011) and the host galaxy extinction is from the literature of individual events (see Pritchard et al. 2014 and references therein). In the database itself, each event includes a reference field listing relevant publications. In the correlation program we use the extinction formula of Cardelli et al. (1989) with a default value of $R_{v}=3.1$ as most of the $E(B-V)$ values available are Galactic, but $R_{v}$ is an adjustable parameter, $E(B-V)$ values can be adjusted, and the extinction law of Calzetti et al. (2000) is also an option. Thus, the user may choose to use the best known values for extinction or enter other values. As an example of the code output, Figure 2 shows Swift observations with a set of simple physics LANL analytical models described above. These models have a $25 M_{\odot}$ progenitor star, nickel mass of $0.1 M_{\odot}$ a radius of $10^{12} \mathrm{~cm}$, and a temperature of 80 million $\mathrm{K}$. The variable parameters are the ejecta mass $\left(M_{\mathrm{Ej}}=4\right.$ or $\left.8 M_{\odot}\right)$, the explosion energy ( 1 or 6 foe), and the density profile $\left(\rho \propto r^{0}\right.$ or $r^{-2.5}$ ).

The code calculates a least-squares minimization for each filter and the sum of all filters by comparing the model with a Gaussian (or polynomial) fit of the observation. An added feature to the correlation code is the ability to shift the models in both magnitude and time interactively. There can be ambiguity in the explosion time ${ }^{8}$ and in the distance of the $\mathrm{SN}$ or if one wants to determine how to better fit a new model from an existing model. This ability to shift the models in time/ magnitude space allows for some manual fitting rather than relying solely on automatic fits. For each interactive shifting, the resulting least-squares-fitted values are output to a commadelimited file.

However, a simple least-squares or similar fitting to determine the goodness-of-fit is not necessarily the best method for determining how a model does or does not fit. Knowing where the model does not fit the data gives insight into producing better models. For example, a model may have the same time of peak brightness and fall-off slope from the peak as an observation, but be too bright overall, missing the observational data points. On the other hand, a straight line

\footnotetext{
8 We note that in our database, time $=0$ is defined as the shock breakout time.
}

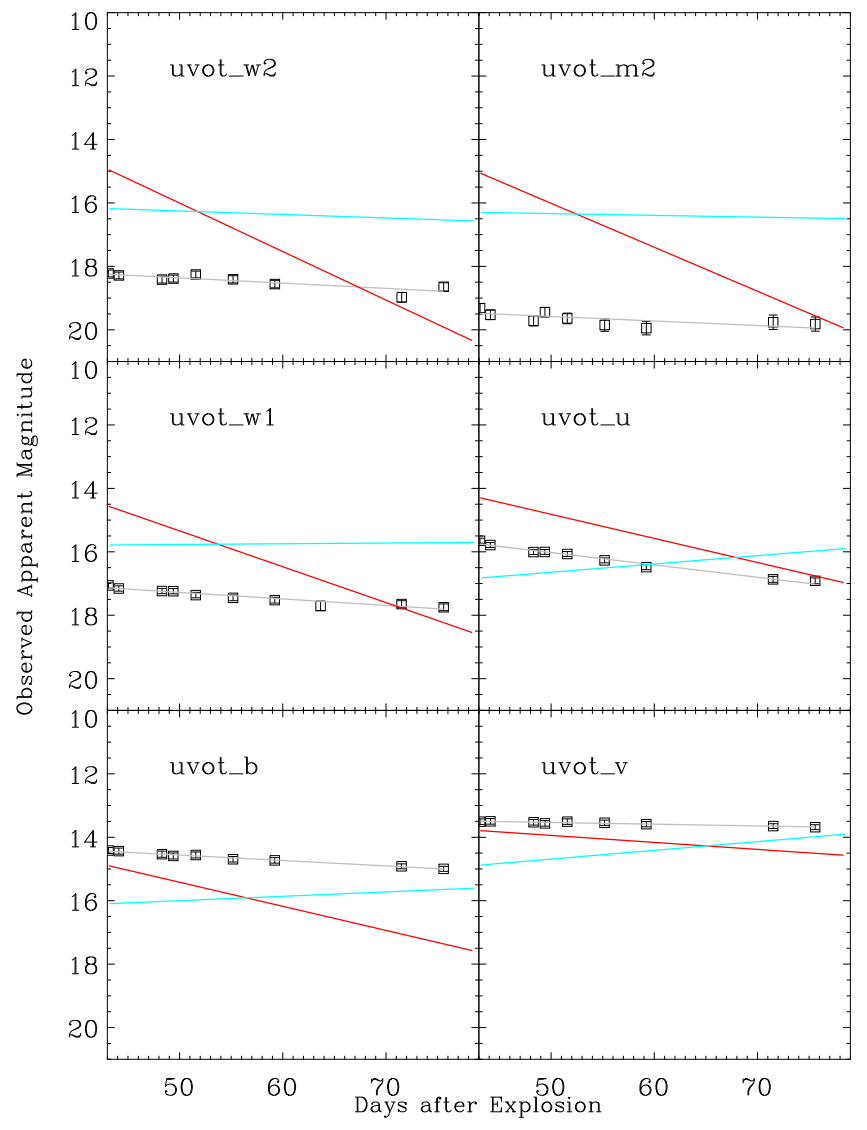

Figure 3. Example of the slope-fitting correlation late-time tail from day 43 to 80. The open squares are SN 2012aw, a type IIP, from the optical Swift-UVOT band passes. The gray line is the linear fit to the observations The red line and cyan line are linear fits to the models in Figure 2. The slope fitting gives this more detailed diagnostic to produce better models.

may pass through some data points, but this model would not be representative of the underlying physics of the SN. A simple least-squares analysis would identify the straight line as the better fit even if the other model was a better representation. A good light curve fit for a transient stems from modeling physical processes relevant in the SN emission. Thus, we have focused on the slope because, in many cases, it provides a cleaner diagnostic of the nature of the progenitor. The slope of the light curve is increasingly being used by theorists and observers to differentiate supernovae. In type Ia $\mathrm{SNe}$, the slope and the change in the slope can be used to pinpoint the timing of the SN (Arnett et al. 2016). The slope of the plateau decay in IIp $\mathrm{SNe}$ has also been used to determine the properties of the SN explosion (Anderson et al. 2014; Dhungana et al. 2016; Silverman et al. 2017).

Thus, the second correlation option compares the slopes within a certain user-specified time region. We account for distance and extinction in the observations in the same way as described above. We also include interactive fitting in both time and magnitude as before. The slopes of the observations and models within the selected time region are calculated using a simple linear fit. We leave to the user the case of specifying a region that is short enough to be described by a line, yet contains data points of interest. As an example, Figure 3 shows the late-time tail of SN 2012aw and the red and cyan models from Figure 2. These models have $E=1$ foe and $\rho \propto r^{0}$ with the red model having $M_{\mathrm{Ej}}=4 M_{\odot}$ and the cyan having 


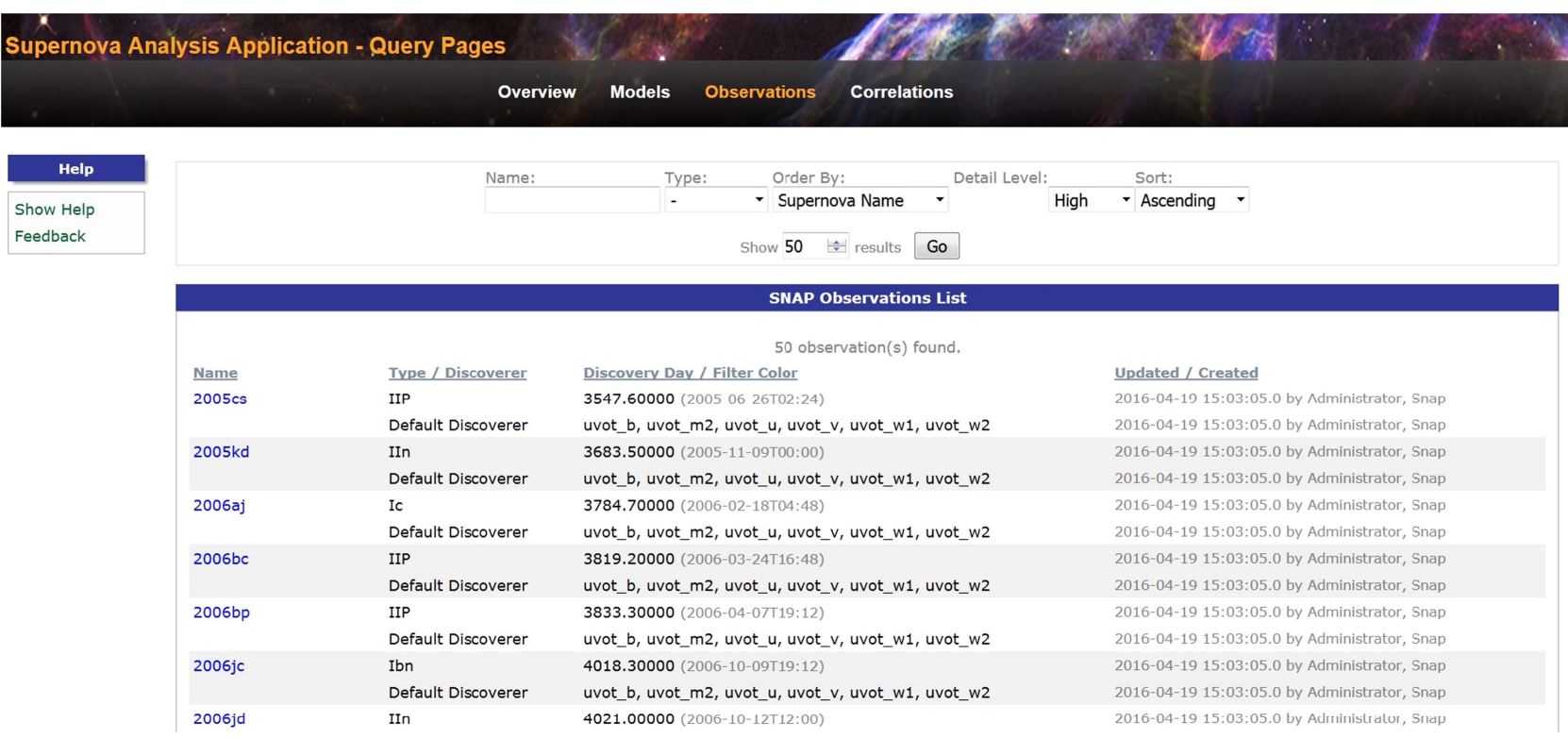

Figure 4. Screen capture of the observation query page. Users can search for SN names by year, or type. Each SN is a hyperlink that gives more detailed information, including distance, extinction, dates, coordinates, etc., and light curve information. The hyperlinks also cross-reference any correlations that used that SN.

$M_{\mathrm{Ej}}=8 M_{\odot}$. These represent a bounding region for this model as the red model is too steep and the cyan is not steep enough. Thus, the slope fitting gives a more detailed diagnostic to produce better models. The slopes of the observations and model are printed to a comma-delimited file for user comparison.

Theoretically, the models in the database are not intended to match any one event, but to be representative of a family of SN types. The power of SNAP as an analysis tool is the ability to see not only what model-observation pairs work but, even more importantly, which ones do not. These types of correlations between models and observations give us rapid feedback on what we are understanding and what physics we are missing in the models. The SNAP database is, of course, a perpetual work in progress. The goal is to have a database containing models covering every type of CCSNe (and eventually Ias) with reasonable parameter space coverage.

\section{The Web Application}

SNAP is accessed through a web application hosted at SwRI. ${ }^{9}$ From the web, users can download observations or model light curves and view correlations. Current upload capability can be done through contact with the PI (A. Bayless), but will be available via the web application in the next version of SNAP. Correlations are one-to-many relations, but certain filters on the correlation can be used. For example, a model-to-observation correlation may only include observations of SNe IIP and not all types. An observation-to-model correlation may only include models within a certain progenitor mass range, for instance. When users upload their own observations or models and run their own correlations, they will have the option of saving them to the database, bypassing the current interface with the PI. Permanent correlation additions will be cross-linked to the models and the observations, and appear on the respective model and observation pages with the entry. For security, users will

\footnotetext{
9 The form to request access to the full SNAP system is available at snap. space.swri.edu. Access is granted via a VPN account.
}

have editing ability on their data or models only, but will be able to view resulting model light curves (not source code) from other users, all telescope observations, which are public domain, and all correlations that have been run.

The observational page has the options to search for a particular SN (with wildcard options, e.g., 2012* returns all $\mathrm{SNe}$ from 2012) or filter by a particular SN type. Figure 4 shows a screen capture from the observation query page. The high level returns the discovery date, MJD and calendar day, and the available filter band passes that have stored observations. Figure 5 shows the entry for SN 2012aw including the plotted light curves. Clicking on the light curve plot allows users to download a text file of the light curves giving JD, magnitude, and error in magnitude if available. The model page has the options to search for a range of progenitor star masses, the creator/research group, the SN type, and how mass was removed for Type $\mathrm{IIb} / \mathrm{Ib} / \mathrm{Ic}$. Figure 6 shows a screen capture from the model query page. The correlation page allows users to compare observations against a set of models, or a model against a set of observations. Figure 7 shows a screen shot from the model-to-observation correlation query page. On this page there are options to search by correlation creator, status, SN type, and when the correlation was made. The same options exist on the observation-to-model page (Figure 8), but also have a filter by observational discovery day or shock breakout day (if known). Any correlations that are saved between models and observations are cross-linked to the model on the model page and the observation on the observation page. Thus, when searching for a particular model or observation, if there is a saved correlation, it will also be displayed in the search on those respective pages.

\section{Conclusion}

The era of large SN surveys will provide the opportunity to statistically study $\mathrm{SNe}$ in unprecedented numbers. Large surveys will also capture rare, exotic events which could contribute meaningfully to our understanding of the as-yet enigmatic CCSN mechanism, which must be quickly identified 


Name:
Created:
Updated:
Reviewed:
Type:
Galactic E(B-V):
Filter Color:
Distance (Mpc):
Distance Uncertainty (Mpc):
Distance Modulus:
Distance Modulus Uncertainty:
Host E(B-V):
Upper Limit Day:
Shock Breakout Day:
Shock Breakout Day Uncertainty:
Discoverer:
Discovery Day:
Notes:
References:

2012aw

2016-10-10 22:09:00.0 by Administrator, Snap

2017-08-15 22:04:29.0 by Administrator, Snap

IIP

\subsection{4}

uvot_b, uvot_m2, uvot_u, uvot_v, uvot_w1, uvot_w2

10.11

0.9

30.02

0.2

$6001.80000(2012-03-15 T 07: 12: 00)$

$6002.60000(2012-03-16 T 02: 24: 00)$

0.8

Default Discoverer

6003.40000 (2012-03-16T21:36:00)

Quadri, U., Strabla, L., Girelli, R., et al. 2012, CBET, 3054, 1

Bayless, A. J., Pritchard, T. A., Roming, P. W. A., et al. 2013, ApJL, 764, L13

\section{Light Curve Data Sets (click on chart to download data as text file)}

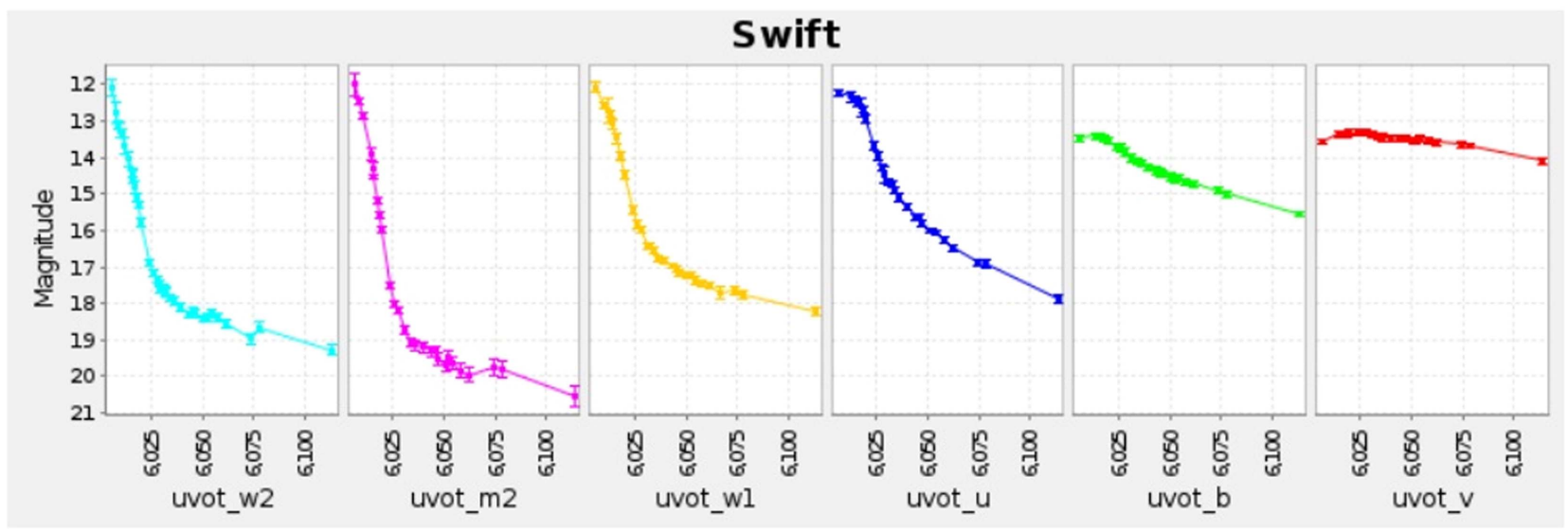

Figure 5. Screen capture of the observation page for SN 2012aw. The page shows the available information and light curves, which can be downloaded as a text file by clicking on the light curve plot.

among hundreds of other $\mathrm{SNe}$ for follow-up. To study the broad spectrum of SN events in this flood of data, a means of rapidly and robustly characterizing transients against highfidelity SN models is required. Additionally, the rapid improvements in computing are making more sophisticated models possible, which then require a means to validate these models.

In this paper, we have presented SNAP, a user-friendly database of SN observations and models with a novel fitting scheme which automates this process, accessed through a web browser. The database has utility for studies of single events, allows for comparisons of observations with an array of light curve models or vice versa, as well as large data sets of SN observations characteristic of upcoming surveys. We invite members of the community to contribute and use this database for their research needs. Indeed, the real power of SNAP is the cumulative "network effect" of having users add models and data to the database through the web application-as more models and data are added, more correlations will be possible, giving more insight into SN behavior. SNAP will allow near real-time SN light curve fitting, eliminating in many instances the need to model events individually, and will assist observers in identifying the most interesting transients worthy of extended observations.

The authors would like to thank Daniel van Rossum for his work on the SuperNu code. This work was supported by the Southwest Research Institute Internal Research program (Project Number R8333 and R8498) and is supported by the NASA Astrophysical Data Analysis Program (NNH15ZDA001N-ADAP).

\section{Appendix Gaussian Process Using Effective Luminosity}

For a magnitude, $M$, that is a function of time, an effective luminosity $L$, may be introduced such that

$$
M=M_{\min }-2.5 \log _{10}(L)
$$



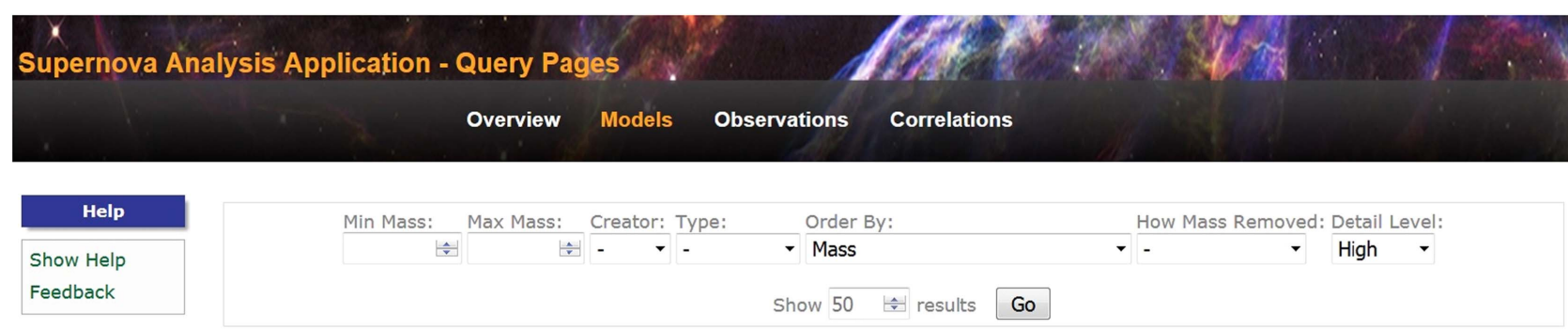

\begin{tabular}{|c|c|c|c|}
\hline \multicolumn{4}{|c|}{ SNAP Model List } \\
\hline \multicolumn{4}{|c|}{$\begin{array}{l}50 \text { model(s) found. } \\
\text { Model Approach Name / }\end{array}$} \\
\hline Model Name (LANL) [CC] & $16 \mathrm{M} 12.6513$ & 16.0 & \\
\hline & $6.27555 E 51$ & 12.6513 & 2016-04-19 15:04:15.0 by Administrator, Snap \\
\hline & 1.0 & Bulk Removal & 2016-04-19 15:04:15.0 by Administrator, Snap \\
\hline Model Name (LANL) [CC] & $16 \mathrm{M} 12.6513$ & 16.0 & \\
\hline & $6.27555 \mathrm{E} 51$ & 12.6513 & 2016-04-19 15:04:15.0 by Administrator, Snap \\
\hline & 1.0 & Bulk Removal & 2016-04-19 15:04:15.0 by Administrator, Snap \\
\hline Model Name (LANL) $[\mathrm{CC}]$ & $16 \mathrm{M} 12.6513$ & 16.0 & \\
\hline & $6.27555 \mathrm{E} 51$ & 12.6513 & 2016-04-19 15:04:15.0 by Administrator, Snap \\
\hline & 1.0 & Bulk Removal & 2016-04-19 15:04:15.0 by Administrator, Snap \\
\hline Model Name (LANL) $[C C]$ & $16 \mathrm{M} 12.6513$ & 16.0 & \\
\hline & $6.27555 \mathrm{E} 51$ & 12.6513 & 2016-04-19 15:04:15.0 by Administrator, Snap \\
\hline & 1.0 & Bulk Removal & 2016-04-19 15:04:15.0 by Administrator, Snap \\
\hline Model Name (LANL) $[\mathrm{CC}]$ & $16 \mathrm{M} 12.6513$ & 16.0 & \\
\hline & $6.27555 \mathrm{E} 51$ & 12.6513 & 2016-04-19 15:04:15.0 by Administrator, Snap \\
\hline & 1.0 & Bulk Removal & 2016-04-19 15:04:15.0 by Administrator, Snap \\
\hline Model Name (LANL) [CC] & $16 \mathrm{M} 12.6513$ & 16.0 & \\
\hline & $6.27555 E 51$ & 12.6513 & 2016-04-19 15:04:15.0 by $\wedge$ dministrator, Snap \\
\hline
\end{tabular}

Figure 6. Screen capture of the model query page. Users can search for models by progenitor mass range, type, and research group (creator of the model). Each model is a hyperlink that gives more detailed information about the model parameters and light curves. The hyperlinks also cross-reference any correlations that used that model.

where $M_{\min }$ is the minimum observed magnitude (the brightest point) of the light curve. Equation (1) is meant to apply to either absolute or apparent magnitude, for any band (since $L$ is chosen to give $M$ ). When $M=M_{\min }$, Equation (1) gives $L=$ $L_{\max }=1$.

The objective is to fit $L$ and the associated error with a Gaussian process, and convert the resulting mean and variance curves back to magnitudes. Scaling the effective peak luminosity to 1 will be useful for the particular Gaussian process described here.

For an error magnitude of $\delta M$, upper and lower bounds can be obtained on the effective luminosity,

$$
M \mp \delta M=M_{\min }-2.5 \log _{10}\left(L^{ \pm}\right),
$$

where $L^{+}\left(L^{-}\right)$is the upper (lower) effective luminosity bound. Consequently, conservatively the error in effective luminosity is

$$
\delta L=\max \left\{L^{+}-L, L-L^{-}\right\}=L^{+}-L=L\left(10^{\delta M / 2.5}-1\right) .
$$

Note that the selection of 2.5 as the coefficient of the base 10 logarithm gives $\delta L / L \sim \delta M$ for $M \sim M_{\min }$ and $\delta M \lesssim 1$. Incorporating the observational noise in the Gaussian process and covariance matrix is now straightforward, since the error can be normalized directly with the effective luminosity, as shown in Equation (7) below.
Suppose there are vectors of $N$ observed magnitudes and errors: $\boldsymbol{M}, \boldsymbol{\delta} \boldsymbol{M}$. Moreover, suppose there is a corresponding set of times: $\boldsymbol{t}$. With Equations (1)-(3) above, these furnish vectors of effective luminosity and error: $\boldsymbol{L}, \boldsymbol{\delta} \boldsymbol{L}$. These effective luminosity vectors will serve as input to the Gaussian process fitting. We follow the standard prescription described in Rasmussen \& Williams (2006). A Gaussian process can be thought of as a stochastic process with random numbers being sampled from an infinite-dimensional Gaussian distribution. This always represents a subset of sampling points from a Gaussian distribution in function space. This function space sampling can be expressed as:

$$
L(t) \sim \mathcal{N}\left(\bar{L}(t), K\left(t, t^{\prime}\right)\right)
$$

where $L$ is a function (of $t$ ) sampled from a Gaussian (or normal, hence symbol $\mathcal{N}$ ) distribution with mean function $\bar{L}$ and covariance function $K$. The multivariate Gaussian for the $N$ observation points is extracted from Equation (4) as

$$
\boldsymbol{L} \sim \mathcal{N}(\overline{\boldsymbol{L}}(\boldsymbol{t}), \boldsymbol{K}(\boldsymbol{t}, \boldsymbol{t})),
$$

where $\overline{\boldsymbol{L}}$ is a vector of mean effective luminosities and $\boldsymbol{K}$ is the covariance matrix with elements $(\boldsymbol{K})_{i, j}=K\left(t_{i}, t_{j}\right)$. The mean is now a vector (of size $N$ ) of mean values, and the covariance is now a matrix (of size $N \times N$ ).

The next assumption (consistent with the Gaussian process framework) is to assume the observed error is also sampled 

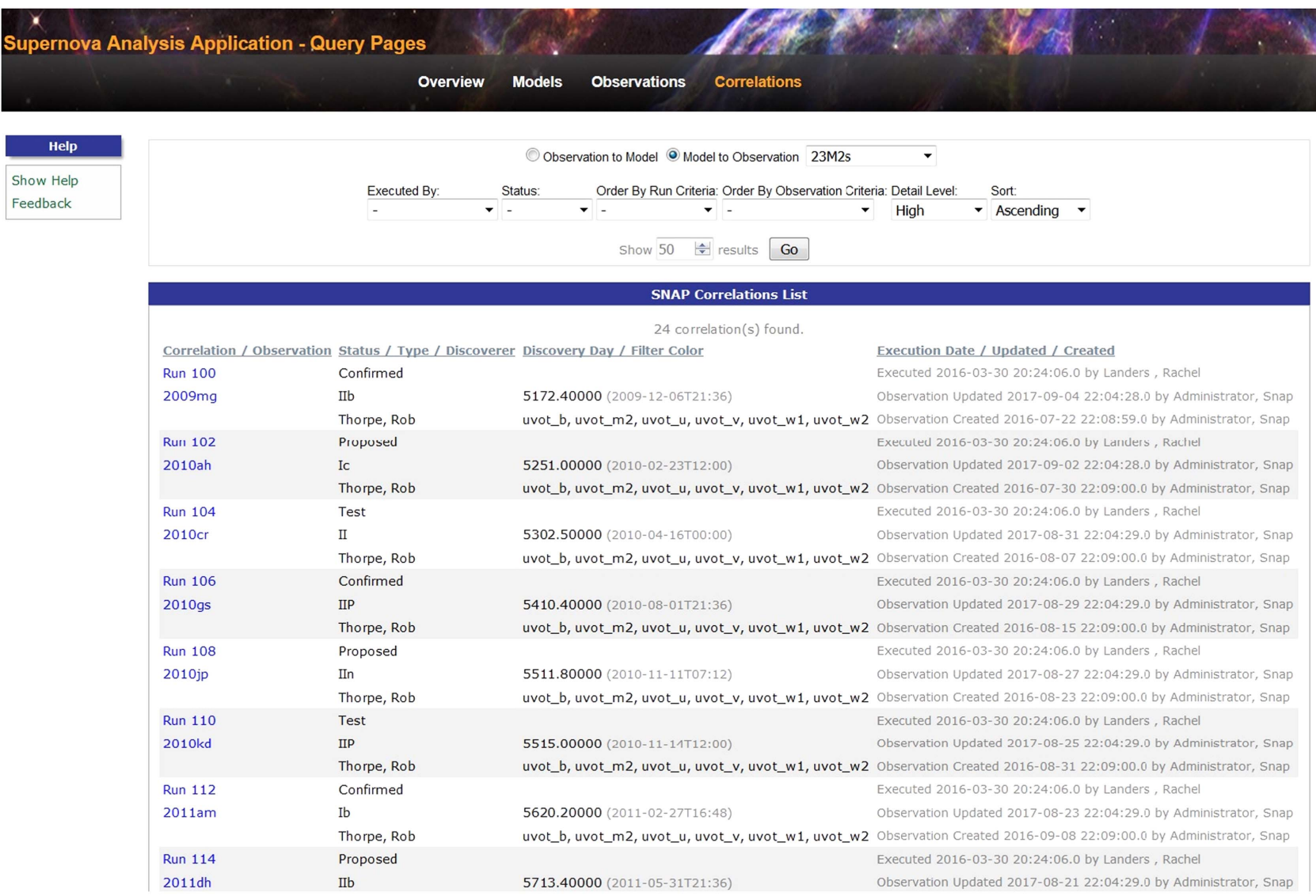

Figure 7. Screen capture of the model-to-observation (M2O) Query page. Users can search for M2O correlations by creator of the correlation or status and ordered by several criteria options (date, SN name, status, etc.). Each correlation is a hyperlink that gives cross-linked information about the model's parameters and the SN observations that were correlated, and information about the correlation.

from a multivariate Gaussian distribution,

$$
\frac{\boldsymbol{\delta} \boldsymbol{L}}{\boldsymbol{L}} \sim \mathcal{N}\left(0, \sigma_{o}^{2} \boldsymbol{I}\right),
$$

where $\boldsymbol{I}$ is the identity matrix and $\sigma_{o}$ is the true standard deviation for the noise of the observation. For the calculations here, the estimator

$$
\hat{\sigma_{o}^{2}}=\frac{1}{N} \sum_{i=1}^{N}\left(\frac{\delta L_{i}}{L_{i}}\right)^{2},
$$

is used in place of $\sigma_{o}^{2}$.

For the covariance function $K$, the squared exponential kernel is assumed,

$$
K\left(t, t^{\prime}\right)=e^{-\left|t-t^{\prime}\right| / 2 \tau^{2}},
$$

where $\tau$ is the so-called "hyperparameter," which is adjusted to optimally fit the observed data. For the case of light curves, this parameter is the characteristic timescale over which points should co-vary. To obtain $\tau$, the log-marginal likelihood is maximized,

$$
\begin{aligned}
\log ( & (\boldsymbol{L} \mid))-\frac{1}{2} \boldsymbol{L}^{T} \boldsymbol{K}(\boldsymbol{t}, \boldsymbol{t})^{-1} \boldsymbol{L} \\
& -\log (\operatorname{det}(\boldsymbol{K}(\boldsymbol{t}, \boldsymbol{t})))-\frac{N}{2} \log (2 \pi),
\end{aligned}
$$

by evaluating for a grid of possible $\tau$ values from 1 to 50 days (roughly the smallest to largest possible covariance measure for a light curve spanning 50 days).

It remains to select the desired prediction points, $t^{*}$ (of size $N^{*}$ ), and calculate the Gaussian process fit at these points. The formulae for the mean and variance of the fit are obtained from the following matrix equations,

$$
\overline{\boldsymbol{L}}^{*}\left(\boldsymbol{t}^{*}\right)=\boldsymbol{K}\left(\boldsymbol{t}^{*}, \boldsymbol{t}\right)\left(\boldsymbol{K}(\boldsymbol{t}, \boldsymbol{t})+{\hat{\sigma_{o}^{2}}}^{\boldsymbol{I}}\right)^{-1} \boldsymbol{L},
$$

and

$$
\begin{aligned}
& V^{*}\left(t^{*}, t^{*}\right)=\boldsymbol{K}\left(\boldsymbol{t}^{*}, \boldsymbol{t}^{*}\right)+\hat{\sigma_{o}^{2}} \boldsymbol{I}-\boldsymbol{K}\left(\boldsymbol{t}^{*}, \boldsymbol{t}\right) \\
& \quad \times\left(\boldsymbol{K}(\boldsymbol{t}, \boldsymbol{t})+\hat{\sigma_{o}^{2}} \boldsymbol{I}\right)^{-1} \boldsymbol{K}\left(\boldsymbol{t}, \boldsymbol{t}^{*}\right),
\end{aligned}
$$

respectively, where $\boldsymbol{K}(\boldsymbol{x}, \boldsymbol{y})$ is the covariance matrix of size (size of $\boldsymbol{x}$ ) $\times$ (size of $\boldsymbol{y}$ ). The fit is obtained by calculating the covariance kernels in Equations (10) and (11) with the value of $\tau$ that maximizes Equation (9). To obtain standard deviations at the prediction points $\left(t^{*}\right)$, the mean luminosity vector is multiplied by the square root of the diagonal of $V^{*}\left(t^{*}, t^{*}\right)$.

$$
\left(\boldsymbol{\delta} \boldsymbol{L}^{*}\right)_{i}=\sqrt{\left(\boldsymbol{V}^{*}\right)_{i, i}}\left(\overline{\boldsymbol{L}}^{*}\right)_{i} .
$$



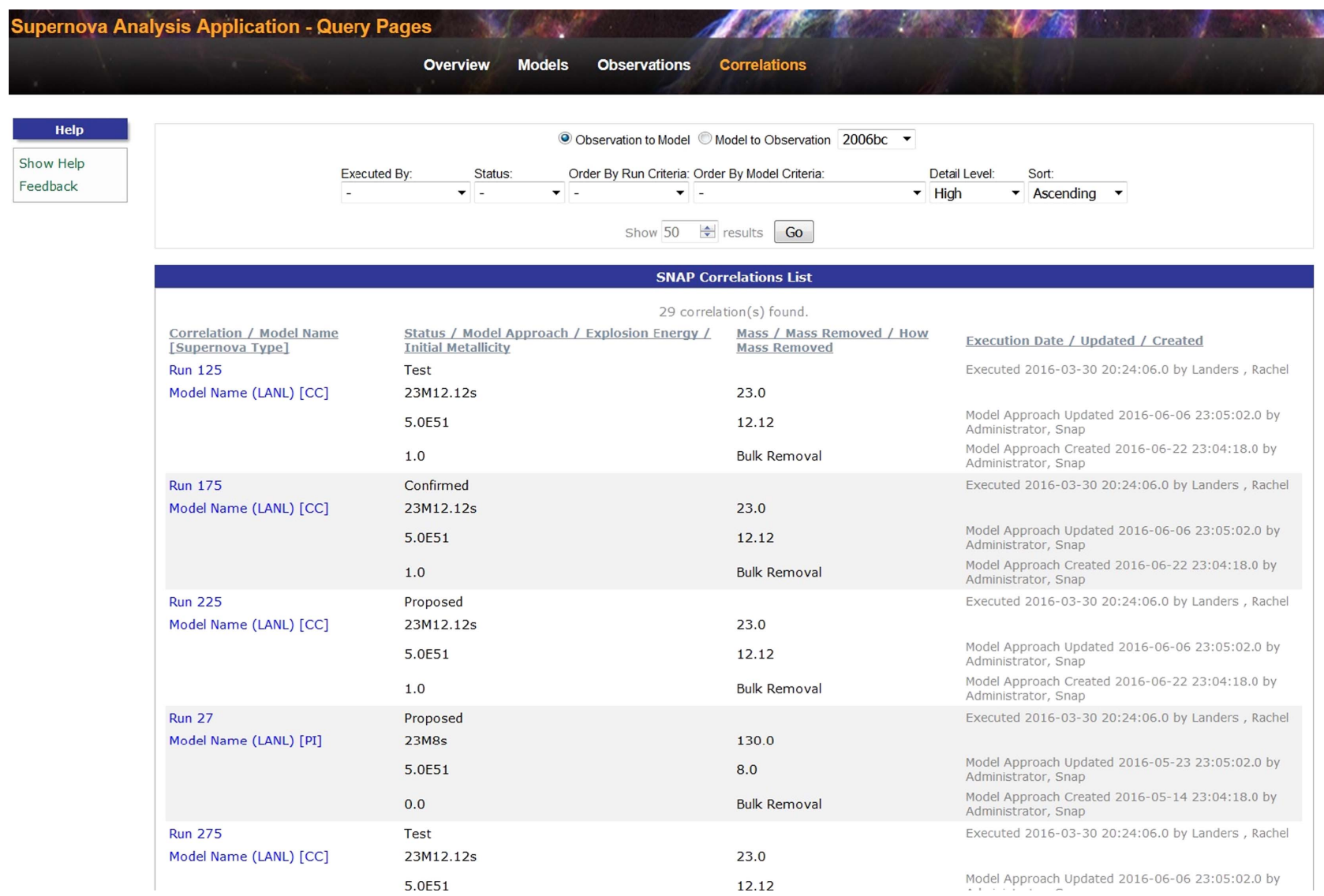

Figure 8. Screen capture of the observation-to-model (O2M) Query page. Users can search for $\mathrm{O} 2 \mathrm{M}$ correlations by creator of the correlation or status and ordered by several criteria options (creation date, SN type, etc.). Each correlation is a hyperlink that gives cross-linked information about the model's parameters and the SN observations that were correlated, and information about the correlation.
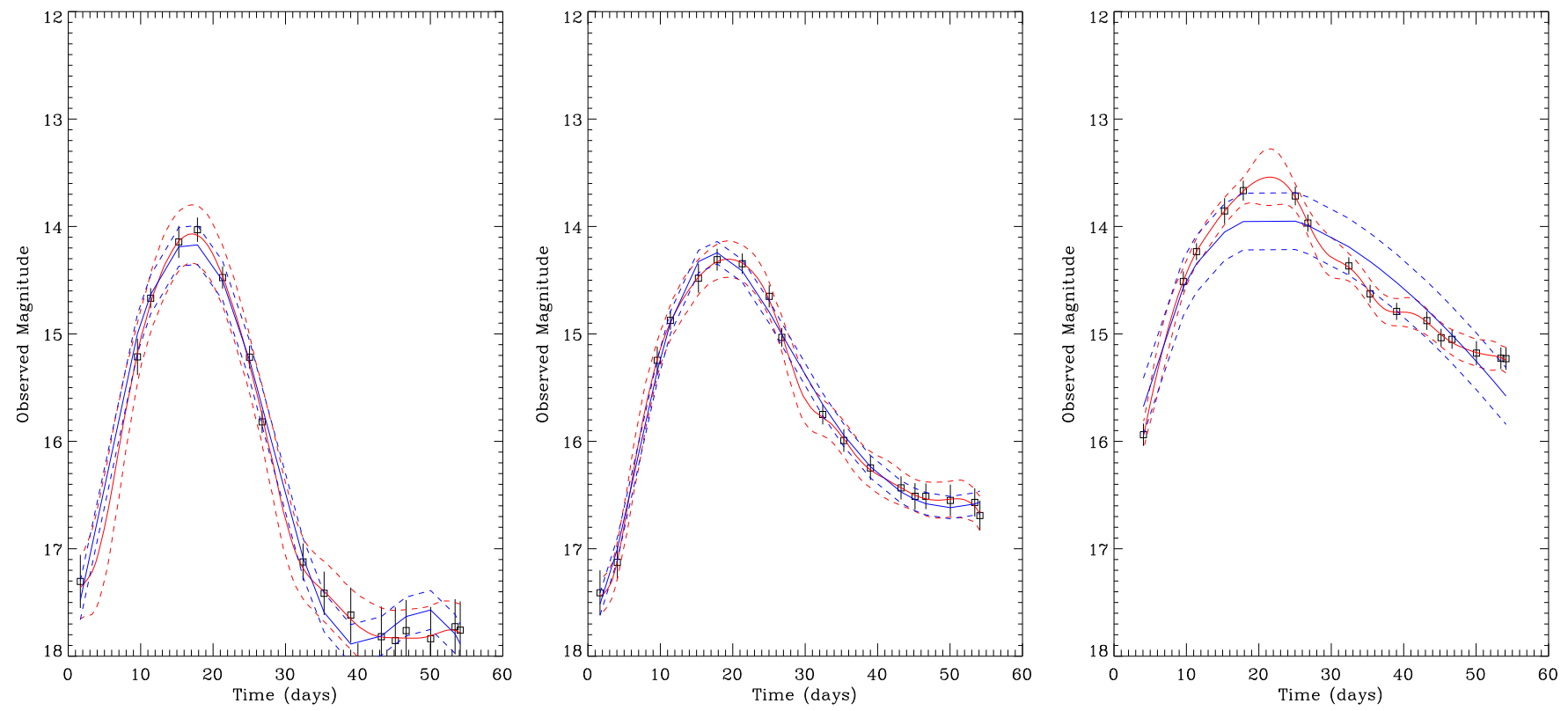

Figure 9. Comparison of Gaussian process to IDL's GAUSSFIT for SN 2008ax in UVOT's u (left), b (middle), and v (right), band passes. The squares are the UVOT observations. The red solid line is the Gaussian process and the red dashed lines are the error in the fit. The blue solid line is the IDL GAUSSFIT result with the blue dashed lines being the error in the fit. For the Gaussian process, $\tau \sim 4$ days in all bands. The discrepancy in the $\mathrm{v}$ band may be due to the lack of a data point at the peak of the fit.

\section{A.1. Comparison to IDL's GAUSSFIT}

In IDL the library function GAUSSFIT preforms a leastsquares fit to the observations using a Gaussian and a quadratic function with three to six degrees of freedom, requiring at least four data points per light curve. This is a slightly different form than the Gaussian only described above, but in testing gives 
similar results. Figure 9 shows the comparision of the two methods for SN 2008ax in UVOT's $u, b$, and v band passes.

\section{ORCID iDs}

Amanda J. Bayless (iD https://orcid.org/0000-0003-0660-5900

Chris L. Fryer (iD https://orcid.org/0000-0003-2624-0056

Brandon Wiggins (iD https://orcid.org/0000-0003-2370-7220

Wesley Even (1D https://orcid.org/0000-0002-5412-3618

Peter W. A. Roming (iD https://orcid.org/0000-0002-

5499-953X

Patrick A. Young (D) https://orcid.org/0000-0003-1705-5991

\section{References}

Abdikamalov, E., Burrows, A., Ott, C. D., et al. 2012, ApJ, 755, 111 Anderson, J. P., González-Gaitán, S., Hamuy, M., et al. 2014, ApJ, 786, 67 Arnett, D. 1996, Supernovae and Nucleosynthesis (Princeton, NJ: Princeton Univ. Press)

Arnett, D., Meakin, C., \& Young, P. A. 2009, ApJ, 690, 1715

Arnett, W. D., Fryer, C. L., \& Matheson, T. 2016, arXiv:1611.08746

Arnett, W. D., \& Meakin, C. 2011, ApJ, 733, 78

Bayless, A. J., Even, W., Frey, L. H., et al. 2015, ApJ, 805, 98

Blinnikov, S. I., \& Popov, D. V. 1993, A\&A, 274, 775

Breeveld, A. A., Landsman, W., Holland, S. T., et al. 2011, in AIP Conf. Ser. 1358, Gamma Ray Bursts 2010, ed. J. E. McEnery, J. L. Racusin, \& N. Gehrels (Melville, NY: AIP), 373

Burns, C. R., Stritzinger, M., Phillips, M. M., et al. 2011, AJ, 141, 19

Calzetti, D., Armus, L., Bohlin, R. C., et al. 2000, ApJ, 533, 682

Cardelli, J. A., Clayton, G. C., \& Mathis, J. S. 1989, ApJ, 345, 245

Chatzopoulos, E., Wheeler, J. C., Vinko, J., et al. 2016, ApJ, 828, 94

de la Rosa, J., Roming, P., Pritchard, T., \& Fryer, C. 2016, ApJ, 820, 74

Densmore, J. D., Thompson, K. G., \& Urbatsch, T. J. 2012, JCoPh, 231, 6925

Densmore, J. D., Urbatsch, T. J., Evans, T. M., \& Buksas, M. W. 2007, JCoPh, 222, 485

Dessart, L., \& Hillier, D. J. 2005, A\&A, 437, 667

Dessart, L., Hillier, D. J., Woosley, S., et al. 2015, MNRAS, 453, 2189

Dhungana, G., Kehoe, R., Vinko, J., et al. 2016, ApJ, 822, 6
Filippenko, A. V. 1997, ARA\&A, 35, 309

Filippenko, A. V. 2005, in ASP Conf. Ser. 332, The Fate of the Most Massive Stars, ed. R. Humphreys \& K. Stanek (San Francisco, CA: ASP), 33

Fleck, J. A., Jr., \& Cummings, J. D. 1971, JCoPh, 8, 313

Frey, L. H., Even, W., Whalen, D. J., et al. 2013a, ApJS, 204, 16

Frey, L. H., Fryer, C. L., \& Young, P. A. 2013b, ApJL, 773, L7

Fryer, C., Benz, W., Herant, M., \& Colgate, S. A. 1999, ApJ, 516, 892

Fryer, C. L., Rockefeller, G., \& Warren, M. S. 2006, ApJ, 643, 292

Gehrels, N., Chincarini, G., Giommi, P., et al. 2004, ApJ, 611, 1005

Gittings, M., Weaver, R., Clover, M., et al. 2008, CS\&D, 1, 015005

Grefenstette, B. W., Harrison, F. A., Boggs, S. E., et al. 2014, Natur, 506, 339

Guillochon, J., Parrent, J., \& Margutti, R. 2017, ApJ, 835, 64

Heger, A., Fryer, C. L., Woosley, S. E., Langer, N., \& Hartmann, D. H. 2003, ApJ, 591, 288

Herant, M., Benz, W., Hix, W. R., Fryer, C. L., \& Colgate, S. A. 1994, ApJ, 435, 339

Kasen, D., Thomas, R. C., \& Nugent, P. 2006, ApJ, 651, 366

Kozyreva, A., Gilmer, M., Hirschi, R., et al. 2017, MNRAS, 464, 2854

Lamers, H. J. G. L. M., \& Nugis, T. 2002, A\&A, 395, L1

Magee, N. H., Abdallah, J., Jr., Clark, R. E. H., et al. 1995, in ASP Conf. Ser. 78 , Astrophysical Applications of Powerful New Databases, ed. S. J. Adelman \& W. L. Wiese (San Francisco, CA: ASP), 51

Meakin, C. A., \& Arnett, D. 2007, ApJ, 667, 448

Poole, T. S., Breeveld, A. A., Page, M. J., et al. 2008, MNRAS, 383, 627

Pritchard, T. A., Roming, P. W. A., Brown, P. J., Bayless, A. J., \& Frey, L. H. 2014, ApJ, 787, 157

Rasmussen, C. E., \& Williams, C. K. I. 2006, Gaussian Processes for Machine Learning (Cambridge, MA: MIT Press)

Roming, P. W., Townsley, L. K., Nousek, J. A., et al. 2000, Proc. SPIE, 4140, 76

Roming, P. W. A., Hunsberger, S. D., Mason, K. O., et al. 2004, Proc. SPIE, 5165,262

Roming, P. W. A., Kennedy, T. E., Mason, K. O., et al. 2005, SSRv, 120, 95

Roming, P. W. A., Pritchard, T. A., Prieto, J. L., et al. 2012, ApJ, 751, 92

Schlafly, E. F., \& Finkbeiner, D. P. 2011, ApJ, 737, 103

Silverman, J. M. Pickett, S., Wheeler, J. C., et al. 2017, MNRAS, 467, 369

Valenti, S., Smartt, S. \& PS1 Science Consortium 2012, MSAIS, 19, 166

Wollaber, A. B. 2015, J. Comput. Theor. Transp., 45, 1

Wollaeger, R. T., \& Van Rossum, D. R. 2014, ApJS, 214, 28

Wollaeger, R. T., van Rossum, D. R., Graziani, C., et al. 2013, ApJS, 209, 36

Young, P. A., \& Arnett, D. 2005, ApJ, 618, 908 